\title{
ITERATIVE PROJECTION METHODS FOR COMPUTING RELEVANT ENERGY STATES OF A QUANTUM DOT
}

\author{
HEINRICH VOSS*
}

Key words. quantum dot, electronic structure, electron states, rational eigenproblem, Schrödinger equation, Arnoldi method, Jacobi-Davidson method, minmax characterization

AMS subject classification. $65 \mathrm{~F} 15,65 \mathrm{~F} 50,81 \mathrm{Q} 10$

Abstract. A computational technique for computing relevant energy levels and corresponding wave functions of an electron confined by a 3D quantum dot embedded in a semiconductor matrix are studied. Assuming an energy and position dependent electron effective mass approximation this problem is governed by a rational eigenvalue problem. We discuss the application of iterative projection method of Arnoldi and Jacobi-Davidson type. Projected problems of small dimension are solved efficiently by safeguarded iteration.

1. Introduction. Semiconductor nanostructures have attracted tremendous attention in the past few years because of their unique physical properties and their potential for applications in micro- and optoelectronic devices. In such nanostructures, the free carriers are confined to a small region of space by potential barriers, and if the size of this region is less than the electron wavelength, the electronic states become quantized at discrete energy levels. The ultimate limit of low dimensional structures is the quantum dot, in which the carriers are confined in all three directions.

In this paper we consider the problem to determine a few relevant energy levels and corresponding wave functions of a three-dimensional quantum dot. Assuming the effective one-band Hamiltonian approximation $H$, the position and energy dependent quasi-particle effective mass approximation, the finite hard wall confinement potential, and the Ben Daniel-Duke boundary condition, this problem is modeled by the Schrödinger equation

$$
H\left(m_{j}\right) u=\lambda u
$$

where the effective electron mass $m_{j}$ is a rational function $m_{j}=f_{j}(\lambda)$ of the energy level for the quantum $\operatorname{dot}(j=q)$ and the matrix surrounding the quantum $\operatorname{dot}(j=m)$.

The nonlinear eigenproblem (1.1) can be solved efficiently by the so called full approximation method $[10,11,12,21]$ if the dimension of a discrete version is not too large (for instance, if the dot is rotational symmetric and (1.1) can be reduced to a two-dimensional problem). However, in the general three-dimensional case this method is too costly, since in every iteration step one has to solve a linear eigenproblem of large dimension.

For sparse linear eigenvalue problems iterative projection methods (Lanczos, Arnoldi, and Jacobi-Davidson method, e.g.) are known to be very efficient. Here approximations to the wanted eigenvalues and eigenvectors are obtained from projections of the eigenproblem of small dimension which are expanded in the course of the algorithm. In $[7,13,15,23]$ this approach was generalized to polynomial eigenproblems and in $[2,17,19,20]$ to the general nonlinear case.

*Institute of Mathematics, Hamburg University of Technology, D-21071 Hamburg, Germany (voss@tu-harburg.de) 
In two recent papers Hwang, Lin, Wang and Wang [8, 9] suggested a numerical method for simulating a three-dimensional pyramidal quantum dot heterostructure. Discretizing the Schrödinger equation (1.1) by a finite volume method one obtains a rational eigenproblem. Multiplying each of the equations by its common denominator one ends up with a polynomial eigenvalue problem of degree 5 , which is solved by a variant of the Jacobi-Davidson method. This approach has two disadvantages: the Schrödinger equation and its rational discretization are symmetric, but this property is not preserved by the polynomial eigenproblem, which makes its numerical solution more involved. Moreover, the wanted smallest positive eigenvalues occur somewhere in the middle of the spectrum of the polynomial problem, which courses some additional numerical problems.

In this paper we make use of the symmetry of problem (1.1) to solve it efficiently. Its eigenvalues can be characterized as minmax values of a Rayleigh functional. This property is inherited by finite element approximations of (1.1), and by projections to small dimensional subspaces which are constructed in iterative projection methods like the Arnoldi and the Jacobi-Davidson method. Hence, these projected problems can be solved efficiently by safeguarded iteration.

Our paper is organized as follows. In Section 2 we state the rational eigenvalue problem which models the electronic behavior of a quantum dot assuming a position and energy dependent quasi-particle effective mass approximation. We rewrite it in variational form, and derive the minimum-maximum characterization of its eigenvalues. Section 3 describes the iterative projection methods, and discusses the solution of the projected rational eigenproblems by safeguarded iteration. Numerical results are given in Section 4 demonstrating the efficiency of the method. Some concluding remarks are given in Section 5 .

2. The governing Schrödinger equation. We consider the problem to compute relevant energy states and corresponding wave functions of a three dimensional semiconductor quantum dot. Let $\Omega_{q} \subset \mathbb{R}^{3}$ be a domain occupied by the quantum dot, which is embedded in a bounded matrix $\Omega_{m}$ of different material. A typical example is an InAs pyramidal quantum dot embedded in a cuboid GaAs matrix.

We consider the one-band envelope-function formalism for electrons and holes in which the effective Hamiltonian is given by

$$
\hat{H}=-\frac{\hbar^{2}}{2} \nabla \cdot\left(\frac{1}{m(\lambda, x)} \nabla\right)+V(x)
$$

where $\hbar$ is the reduced Planck constant, and $\nabla$ denotes the spatial gradient.

Assuming non-parabolicity for the electron's dispersion relation the electron effective mass $m(\lambda, x)$ is constant on the quantum dot and the matrix for every fixed energy level $\lambda$, and is taken as $[1,3]$

$$
\frac{1}{m_{j}(\lambda)}:=\left.\frac{1}{m(\lambda, x)}\right|_{x \in \Omega_{j}}=\frac{P_{j}^{2}}{\hbar^{2}}\left(\frac{2}{\lambda+E_{g, j}-V_{j}}+\frac{1}{\lambda+E_{g, j}-V_{j}+\Delta_{j}}\right), \quad j \in\{m, q\}
$$

where the confinement potential $V_{j}:=\left.V\right|_{\Omega_{j}}$ is piecewise constant, and $P_{j}, E_{g, j}$ and $\Delta_{j}$ are the momentum matrix element, the band gap, and the spin-orbit splitting in the valence band gap for the quantum $\operatorname{dot}(j=q)$ and the matrix $(j=m)$, respectively.

To determine the relevant energy states and corresponding wave functions we 
have to solve the governing Schrödinger equation

$$
-\nabla \cdot\left(\frac{\hbar^{2}}{2 m_{j}(\lambda)} \nabla u\right)+V(x) u=\lambda u, x \in \Omega_{q} \cup \Omega_{m} .
$$

Since the wave function decays outside the quantum dot very rapidly, it is reasonable to assume homogeneous Dirichlet conditions $u=0$ on the outer boundary of $\Omega_{m}$, and on the interface between the quantum dot and the matrix the Ben Daniel-Duke condition [6] holds

$$
\left.\frac{1}{m_{q}} \frac{\partial u}{\partial n_{q}}\right|_{\partial \Omega_{q}}=\left.\frac{1}{m_{m}} \frac{\partial u}{\partial n_{m}}\right|_{\partial \Omega_{m}}, x \in \partial \Omega_{q} \cap \partial \Omega_{m} .
$$

Here $n_{q}$ and $n_{m}$ denote the outward unit normal on the boundary of $\Omega_{q}$ and $\Omega_{m}$, respectively.

Multiplying (2.3) by $v \in H_{0}^{1}(\Omega), \Omega:=\bar{\Omega}_{q} \cup \Omega_{m}$, and integrating by parts one gets the variational form of the Schrödinger equation

$$
\begin{aligned}
a(u, v ; \lambda):= & \frac{\hbar^{2}}{2 m_{q}(\lambda)} \int_{\Omega_{q}} \nabla u \cdot \nabla v d x+\frac{\hbar^{2}}{2 m_{m}(\lambda)} \int_{\Omega_{m}} \nabla u \cdot \nabla v d x+V_{q} \int_{\Omega_{q}} u v d x \\
& +V_{m} \int_{\Omega_{m}} u v d x=\lambda \int_{\Omega} u v d x=: \lambda b(u, v) \quad \text { for every } v \in H_{0}^{1}(\Omega) .
\end{aligned}
$$

For $g_{j}-V_{j}>0, j \in\{m, q\}$ and every $\lambda>0$ the bilinear form $a(\cdot, \cdot ; \lambda)$ is symmetric, bounded, and $H_{0}^{1}$-elliptic, and $b(\cdot, \cdot)$ is bilinear, positive definite, bounded, and completely continuous.

Hence, by the Lax-Milgram lemma the variational eigenproblem (2.5) is equivalent to the nonlinear eigenvalue problem

$$
T(\lambda) u=0
$$

where $T(\lambda): H_{0}^{1}(\Omega) \rightarrow H_{0}^{1}(\Omega), \lambda \geq 0$, is a family of bounded operators. For fixed $\lambda \geq 0$ the linear eigenvalue problem

$$
T(\lambda) u=\mu u
$$

has a countable set of eigenvalues $\mu_{1} \geq \mu_{2} \geq \ldots$ which satisfy a maxmin characterization

$$
\mu_{j}=\max _{\operatorname{dim} V=j} \min _{u \in V, u \neq 0} \frac{\langle T(\lambda) u, u\rangle}{\langle u, u\rangle} .
$$

Here $\langle\cdot, \cdot\rangle$ denotes the usual inner product in $H_{0}^{1}(\Omega)$.

Moreover, for

$$
f(\lambda ; u):=\langle T(\lambda) u, u\rangle=\lambda b(u, u)-a(u, u ; \lambda)
$$

it holds

$$
\lim _{\lambda \rightarrow 0+} f(0 ; u)<0<\lim _{\lambda \rightarrow \infty} f(\lambda ; u)=\infty \quad \text { for every } u \neq 0
$$


and

$$
\frac{\partial}{\partial \lambda} f(\lambda ; u)>0 \text { for every } u \neq 0 \text { and } \lambda \geq 0 .
$$

Hence, for every $u \neq 0$ the real equation $f(\lambda ; u)=0$ has unique solution $p(u)$. This equation therefore defines a functional $p: H_{0}^{1}(\Omega) \backslash\{0\} \rightarrow \mathbb{R}$ which is called Rayleigh functional.

Obviously, if $T(\lambda):=\lambda I-A$ corresponds to a linear eigenvalue problem, then the Rayleigh functional $p$ is just the well known Rayleigh quotient. It is remarkable that nonlinear eigenproblems satisfying the conditions (2.8), (2.10) and (2.11) have similar properties as selfadjoint and completely continuous linear eigenproblems. The nonlinear eigenvalue problem (2.6) has a countable set of eigenvalues which can be characterized as minmax values of the Rayleigh functional $p$ (cf. [5, 22]):

TheOREM 2.1.

(i) The Schrödinger equation (2.3) modelling the quantum dot with electron effective mass $m_{j}$ given in (2.2) has a countable set of eigenvalues

$$
0<\lambda_{1} \leq \lambda_{2} \leq \lambda_{3} \leq \ldots
$$

which all have finite multiplicity, and the only cluster point of which is $\infty$.

(ii) The $k$ :th smallest eigenvalue $\lambda_{k}$ can be characterized as

$$
\lambda_{k}=\min _{\operatorname{dim} V=k} \max _{u \in V, u \neq 0} p(u)
$$

(iii) $\tilde{\lambda}$ is the $k$ :th smallest eigenvalue of (2.3) if and only if $\mu=0$ is the $k$ :th largest eigenvalue of the linear eigenproblem

$$
T(\tilde{\lambda}) u=\mu u
$$

(iv) The minimum in (2.12) is attained for the invariant subspace of $T\left(\lambda_{k}\right)$ corresponding to its $k$ :th largest eigenvalues.

If the Schrödinger equation (2.3) is discretized by a Galerkin method (finite elements, e.g.) one gets a rational matrix eigenvalue problem

$$
S(\lambda) x:=\lambda M x-\frac{1}{m_{q}(\lambda)} A_{q} x-\frac{1}{m_{m}(\lambda)} A_{m} x-B x=0
$$

where

$$
\begin{gathered}
A_{j}=\left(\int_{\Omega_{j}} \nabla \phi_{k} \cdot \nabla \phi_{\ell} d x\right)_{k, \ell}, j \in\{q, m\} \\
M=\left(\int_{\Omega} \phi_{k} \phi_{\ell} d x\right)_{k, \ell} \text { and } B=\left(V_{q} \int_{\Omega_{q}} \phi_{k} \phi_{\ell} d x+V_{m} \int_{\Omega_{m}} \phi_{k} \phi_{\ell} d x\right)_{k, \ell}
\end{gathered}
$$

and $\phi_{k}$ denotes a basis of the ansatz space.

$A_{q}, A_{m}$ and $B$ are symmetric and positive semi-definite, and $M$ is positive definite, and for $\lambda \geq 0$ the matrix

$$
\frac{\hbar^{2}}{2 m_{q}(\lambda)} A_{q}+\frac{\hbar^{2}}{2 m_{q}(\lambda)} A_{q}
$$

is positive definite. Hence, the eigenvalues of the dicretized problem (2.13) satisfy a minmax principle as well, and it follows from the minmax characterization (2.12) of the nonlinear Schrödinger equation that the $k$ :th smallest eigenvalues of the discretized problem (2.13) is an upper bound of the corresponding eigenvalue of problem (2.3). 
3. Iterative projection methods. In this section we consider the problem to compute a few eigenvalues and corresponding eigenvectors at the lower end of the spectrum of a nonlinear eigenproblem

$$
S(\lambda) x=0
$$

where $S(\lambda) \in \mathbb{R}^{n \times n}$ for $\lambda>0$ is a family of large and sparse symmetric matrices, such that for every $x \in \mathbb{R}^{n}, x \neq 0$ and for every $\lambda>0$ it holds

$$
\frac{\partial}{\partial \lambda} x^{T} S(\lambda) x>0
$$

Thus, we consider a discretization (2.13) of the Schrödinger equation (2.3) such that its eigenvalues satisfy a minmax characterization. We stress the fact that we do not assume that $S(\cdot)$ corresponds to an orthogonal projection of (2.7), and its eigenvalues are upper bounds of the corresponding eigenvalues of (2.3).

For linear sparse eigenproblems $S(\lambda)=\lambda B-A$ very efficient methods are iterative projection methods like the Lanczos, the Arnoldi, and the Jacobi-Davidson method, e.g., where approximations to the wanted eigenvalues and eigenvectors are obtained from projections of the eigenproblem to subspaces of small dimension which are expanded in the course of the algorithm.

Essentially two types of methods are in use for linear problems: methods which project the problem to a sequence of Krylov spaces (like the Lanczos or the Arnoldi method), and methods which aim at specific eigenpairs like the Jacobi-Davidson method one at a time. Generalizations to nonlinear problems always have to be of the second type, i.e. the current search space has to be expanded by a direction with high approximation potential for the eigenvector wanted next.

Let $V \in \mathbb{R}^{n \times k}$ be an (orthonormal) basis of a subspace of $\mathbb{R}^{n}$, and assume that $\theta$ is an eigenvalue of the projected eigenvalue problem

$$
V^{T} S(\lambda) V y=0,
$$

$y \in \mathbb{R}^{k}$ is a corresponding eigenvector, and benote by $x:=V y$ the corresponding Ritz vector. Then one step of inverse iteration with initial guess $(\theta, x)$, i.e. the direction

$$
v:=S(\theta)^{-1} S^{\prime}(\theta) x,
$$

is a suitable expansion of the current search space $V$, since inverse iteration is known to converge quadratically to simple eigenvalues, and for symmetric eigenproblems it converges even cubically if the eigenvalue approximation is updated by the Rayleigh functional. Its drawback however is that it is too expensive for large problems since in every iteration step one has to solve a large linear system $S(\theta) v=S^{\prime}(\theta) x$ for $v$ where the system matrix $S(\theta)$ varies in every iteration step.

In the next two subsections we will consider expansions of the search space $V$ which approximate the inverse iteration and which do not have this unpleasant property.

3.1. Arnoldi method. The residual inverse iteration introduced by Neumaier [14] suggests the expansion

$$
v=S(\sigma)^{-1} S(\theta) x,
$$

of the search space $V$ (cf. [17, 19]). Here $\sigma$ is a fixed parameter close to the wanted eigenvalues. 
For a linear eigenproblem $S(\lambda)=A-\lambda B$ this is exactly the Cayley transform with pole $\sigma$ and zero $\theta$, and since $(A-\sigma B)^{-1}(A-\theta B)=I+(\sigma-\theta)(A-\sigma B)^{-1} B$ and Krylov spaces are shift-invariant the resulting projection method expanding $V$ by $v$ is nothing else but the shift-and-invert Arnoldi method.

If the linear system $S(\sigma) v=S(\theta) x$ is too expensive to solve for $v$ we may choose as new direction $v=K^{-1} S(\theta) x$ with $K \approx S(\sigma)$, and for the linear problem we obtain an inexact Cayley transform or a preconditioned Arnoldi method. We therefore call the resulting iterative projection method given in Algorithm 1 nonlinear Arnoldi method, although no Krylov space is constructed and no Arnoldi recursion holds.

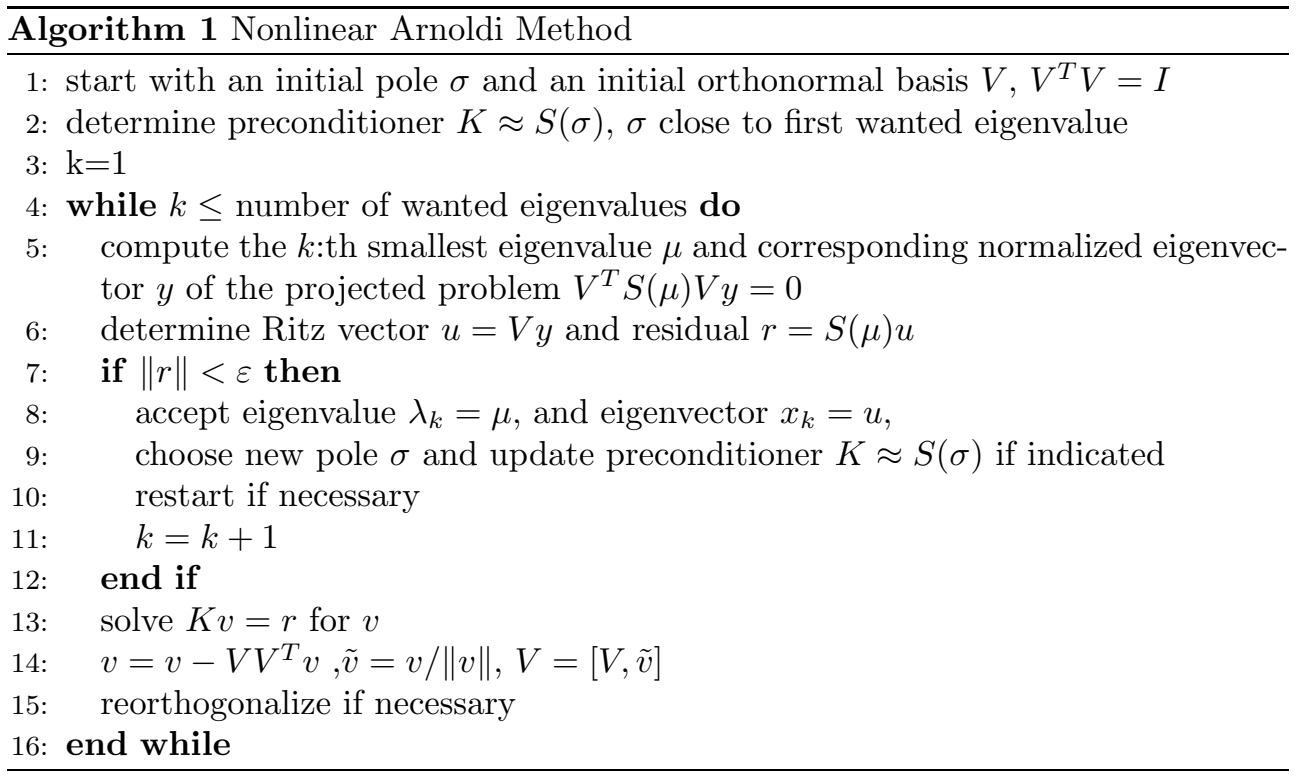

There are many details that have to be considered when implementing the nonlinear Arnoldi method concerning the choice of the initial basis, when and how to update the preconditioner, and how to restart the method. A detailed discussion is given in [19]. Of particular importance is the question how to solve the projected eigenproblem in Step 5: which will be addressed in Subsection 3.3.

3.2. Jacobi-Davidson method. Arnoldi type methods are quite efficient in solving sparse nonlinear eigenproblems if an accurate preconditioner $K \approx S(\sigma)$ is at hand. If this is not the case, then the convergence deteriorates considerably. In this situation Jacobi-Davidson type methods offer an appealing alternative.

A natural generalization of the Jacobi-Davidson method for linear eigenproblems which was already suggested in [15] for polynomial eigenvalue problems and which was studied in [2] and [20] for general nonlinear eigenproblems is the following one: Suppose that the columns of $V \subset \mathbb{R}^{n}$ form an orthonormal basis of the current search space, and let $(x, \theta)$ be a Ritz pair of (3.1) with respect to $V$, i.e. $V^{T} S(\theta) V y=0$, $x=V y$. Then we consider the correction equation

$$
\left(I-\frac{p x^{T}}{x^{T} p}\right) S(\theta)\left(I-\frac{x x^{T}}{x^{T} x}\right) z=-r, \quad z \perp x
$$

where $p:=S^{\prime}(\theta) x$ and $r:=S(\theta) x$. 
Equation (3.6) can be rewritten as $S(\theta) z-\alpha p=-r$, where $\alpha$ has to be chosen such that $z \perp x$. Solving for $z$ we obtain

$$
z=-x+\alpha S(\theta)^{-1} p=-x+\alpha S(\theta)^{-1} S^{\prime}(\theta) x,
$$

and $x=V y$ yields that $\tilde{z}:=S(\theta)^{-1} S^{\prime}(\theta) x \in \operatorname{span}[V, z]$.

Hence, as in the linear case the new search space $\operatorname{span}[V, z]$ contains the vector obtained by one step of inverse iteration with shift $\theta$ and initial vector $x$, and again we may expect quadratic or even cubic convergence of the resulting iterative projection method, if the correction equation (3.6) is solved exactly.

As in the linear case the correction equation does not have to be solved exactly to maintain fast convergence, but usually a few steps of a Krylov solver with an appropriate preconditioner suffice to obtain a good expansion direction of the search space.

The approximate solution of the correction equation has to be orthogonal to $x$. If (3.6) is solved by a Krylov solver and the initial approximation is orthogonal to $x$ then all iterates are orthogonal to $x$ as well.

In the correction equation (3.6), the operator $S(\theta)$ is restricted to map the subspace $x^{\perp}$ into itself. Hence, if $K \approx S(\theta)$ is a preconditioner of $S(\theta)$ then a preconditioner for an iterative solver of (3.6) should be modified correspondingly to

$$
\tilde{K}:=\left(I-\frac{p x^{T}}{x^{T} p}\right) K\left(I-\frac{x x^{T}}{x^{T} x}\right) .
$$

With left-preconditioning, equation (3.6) becomes

$$
\tilde{K}^{-1}\left(I-\frac{p x^{T}}{x^{T} p}\right) S(\theta)\left(I-\frac{x x^{T}}{x^{T} x}\right) z=-\tilde{K}^{-1} r, \quad z \perp x .
$$

It was already demonstrated in [16] for linear problems that taking into account the projectors in the preconditioner, i.e. using $\tilde{K}$ instead of $K$ in a preconditioned Krylov solver, raises the cost only slightly. In every step one has to solve one linear system $K w=y$, and to initialize the solver requires only one additional solve. The transfer to (3.7) is straightforward [2].

A template for the nonlinear Jacobi-Davidson method is similar to Algorithm 1. One only has to replace the solve of system $K v=r$ in Step 13: by a few steps of a preconditioned Krylov solver for the correction equation (3.6).

Hwang et al. $[9,8]$ suggested to avoid the iterative solution of the correction equation (3.6) replacing $S(\theta)$ by some preconditioner $K \approx S(\theta)$

$$
\left(I-\frac{p x^{T}}{x^{T} p}\right) K\left(I-\frac{x x^{T}}{x^{T} x}\right) z=-r, \quad z \perp x
$$

which yields the approximate solution of (3.6)

$$
z=-K^{-1} r+\tau K^{-1} p \quad \text { with } \tau=\frac{x^{T} K^{-1} r}{x^{T} K^{-1} p} .
$$

It is interesting to note that this expansion is a linear combination of the Arnoldi expansion $K^{-1} r$ and an approximation $K^{-1} p$ of the direction suggested by inverse iteration. 
3.3. Safeguarded iteration. A crucial point in iterative methods for general nonlinear eigenvalue problems when approximating more than one eigenvalue is to inhibit the method to converge to the same eigenvalue repeatedly. For linear eigenvalue problems this is easy to do by using Schur forms or generalized Schur forms for the projected problem and then locking or purging certain eigenvalues. For nonlinear problems, however, such Schur forms do not exist and this presents one of the most difficult tasks in achieving good convergence.

For symmetric nonlinear eigenproblems satisfying a minmax characterization (2.12) however, its eigenvalues can be computed safely one after the other. The minimum in (2.12) is attained by the invariant subspace of $S\left(\lambda_{k}\right)$ corresponding to the $k$ :th largest eigenvalues, and the maximum by every eigenvector corresponding to the eigenvalue 0 . This suggests the safeguarded iteration for computing the $k$ :th smallest eigenvalue which reads as follows for the projected eigenproblem $P(\lambda) y:=V^{T} S(\lambda) V y=0$ :

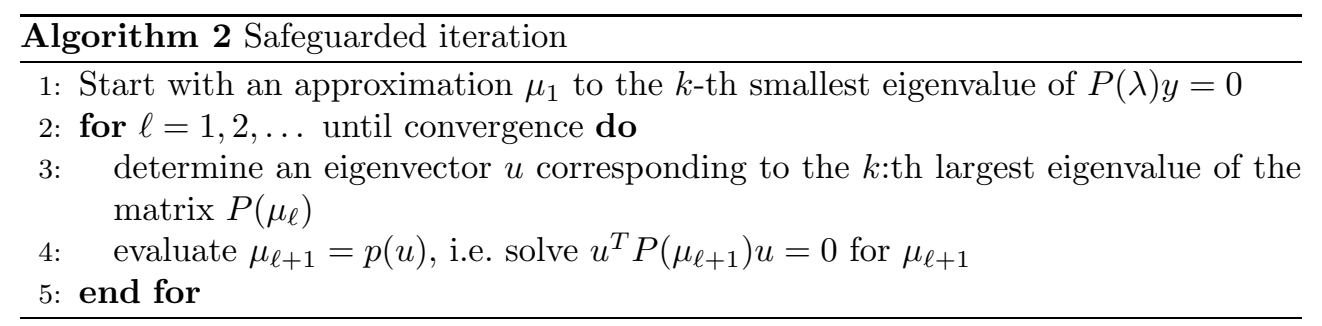

The safeguarded iteration has the following convergence properties [18]: It converges globally to the smallest eigenvalue $\lambda_{1}$. The (local) convergence to simple eigenvalues is quadratic. If $P^{\prime}(\lambda)$ is positive definite, and $u$ in Step 3 of the last algorithm is replaced by an eigenvector of $P\left(\mu_{\ell}\right) u=\mu P^{\prime}\left(\mu_{\ell}\right) u$ corresponding to the $k$ :th largest eigenvalue, then the convergence is even cubic. Moreover, a variant exists which is globally convergent also for higher eigenvalues.

4. Numerical experiments. We consider a pyramidal quantum dot with width $12.4 \mathrm{~nm}$ and height $6.2 \mathrm{~nm}$ embedded in a cubic matrix of size $24.8 \mathrm{~nm} \times 24.8 \mathrm{~nm} \times 18.6$ $\mathrm{nm}$ with the following parameters $P_{q}=0.8503, g_{q}=0.42, \delta_{q}=0.48, V_{q}=0, P_{m}=$ $0.8878, g_{m}=1.52, \delta_{m}=0.34$, and $V_{m}=0.7$. This model was already treated by Hwang, Lin, Wang, and Wang in [9].

The authors of [9] presented a discretization of problem (2.3) by the finite volume method based on a uniform grid which yields a matrix eigenvalue problem (2.13). The occurring matrices $A_{q}, A_{m}$ and $B$ are symmetric and positive semi-definite, $M$ is the identity, and for $\lambda \geq 0$ the matrix

$$
\frac{\hbar^{2}}{2 m_{q}(\lambda)} A_{q}+\frac{\hbar^{2}}{2 m_{q}(\lambda)} A_{q}
$$

is positive definite. Hence, the Arnoldi and the Jacobi-Davidson methods have the properties outlined in Section 3, and the projected eigenproblems can be solved by safeguarded iteration. Notice however, that the discretized problem is not obtained by a projection of problem (2.3) to a finite dimensional space, and therefore the eigenvalues of (2.13) are not guaranteed to be upper bounds of the corresponding eigenvalues of $(2.3)$.

Multiplying each of the equations by its common denominator Hwang et al. transformed (2.13) to a polynomial eigenvalue problem of degree 5 which they solved by 
TABLE 4.1

Uniform finite volume discretization

\begin{tabular}{|r|r|r|r|r|r|}
\hline $\operatorname{dim}$ & $\lambda_{1}$ & $\lambda_{2 / 3}$ & $\lambda_{4}$ & $\lambda_{5}$ & CPU time \\
\hline $2^{\prime} 475$ & 0.41195 & 0.58350 & 0.67945 & 0.70478 & $0.68 \mathrm{~s}$ \\
$22^{\prime} 103$ & 0.40166 & 0.57668 & 0.68418 & 0.69922 & $8 \mathrm{~s}$ \\
$186^{\prime} 543$ & 0.39878 & 0.57477 & 0.68516 & 0.69767 & $151 \mathrm{~s}$ \\
$1^{\prime} 532^{\prime} 255$ & 0.39804 & 0.57427 & 0.68539 & 0.69727 & $4018 \mathrm{~s}$ \\
$12^{\prime} 419^{\prime} 775$ & 0.39785 & 0.57415 & & & overnight \\
\hline
\end{tabular}

the variant of the Jacobi-Davidson method mentioned at the end of Subsection 3.2. This approach has two disadvantages: Symmetry properties of the rational eigenproblem are destroyed, which makes its numerical solution more involved. Moreover, the wanted smallest positive eigenvalues occur somewhere in the middle of the spectrum of the polynomial problem, which courses some additional numerical problems.

We solved the rational eigenproblem (2.13) directly by the nonlinear Arnoldi method [19] (not taking advantage of the fact that the finite difference stencils are identical for all discretization points in the matrix and in the quantum dot, respectively) under MATLAB 7.0.4 on an AMD Opteron processor with 4 GByte RAM and $2.2 \mathrm{GHz}$. Table 4.1 contains the approximations to the smallest 5 eigenvalues and the CPU times. It is evidentthat there is a significant difference between our calculations and the corresponding results in [9], despite the fact that the parameters in our calculations and in [9] were identical. This possibly could be attributed to calculation errors in [9].

The uniform grid is not appropriate for discretizing (2.5) since the wave functions corresponding to small energy levels are mainly concentrated on the quantum dot and decay rapidly outside, whereas the volume occupied by the quantum dot is only less than $3 \%$ of $\Omega$.

Using FEMLAB [4] we discretized (2.5) by cubic Lagrangian elements on a tetrahedral grid with $96^{\prime} 640$ degrees of freedom such that 43 ' 615 DoFs where located in the quantum dot, 43'897 DoFs in the matrix, and 9'128 DoFs on the interface. We solved the rational eigenproblem by the nonlinear Arnoldi method, the Jacobi-Davidson method, and the variant of Hwang et al.

We started the methods with a constant vector on $\bar{\Omega}_{q} \cup \Omega_{m}$ which is far away from an eigenvector, and we terminated the iteration for an eigenvalue, if the residual norm was less than $10^{-8}$. Table 4.2 contains the approximations to the smallest 5 eigenvalues, the number of iterations to obtain the approximations, and the CPU times. Notice, that in this case by Theorem 2.1 one gets upper bounds of the corresponding eigenvalues of problem (2.3). Hence, the approximations to $\lambda_{j}$ for $j=1,2,3$ are definitely better than the ones obtained by the finite volume method with more than 12 million DoFs.

The second eigenvalue is a double one. None of the methods had problems to compute it, and to deliver a basis of the eigenspace.

To demonstrate that the Jacobi-Davidson method is more robust than the Arnoldi method with respect to less accurate preconditioners we solved the rational eigenproblem preconditioning with an incomplete LU factorization with different drop tolerances $\tau$. Table 4.3 contains the CPU times for determining the smallest 5 eigenvalues not considering the time that is needed to compute the LU factorization, which is displayed separately in the last column. 
TABLE 4.2

Finite element discretization

\begin{tabular}{|c|c|c|c|c|c|c|}
\hline $\operatorname{dim}$ & $\lambda_{1}$ & $\lambda_{2}$ & $\lambda_{3}$ & $\lambda_{4}$ & $\lambda_{5}$ & CPU time \\
\hline 96'640 & 0.39779 & 0.57411 & 0.57411 & 0.68547 & 0.69714 & \\
\hline $\begin{array}{r}\text { Arnoldi } \\
\text { JD } \\
\text { Hwang et al. }\end{array}$ & $\begin{array}{r}44 \text { it. } \\
9 \text { it. } \\
45 \text { it. }\end{array}$ & $\begin{array}{r}29 \text { it. } \\
7 \text { it. } \\
9 \text { it. }\end{array}$ & $\begin{array}{r}29 \text { it. } \\
9 \text { it. } \\
5 \text { it. }\end{array}$ & $\begin{array}{r}24 \text { it. } \\
5 \text { it. } \\
24 \text { it. }\end{array}$ & $\begin{array}{r}21 \text { it. } \\
6 \text { it. } \\
21 \text { it. }\end{array}$ & $\begin{array}{l}189 \text { sec. } \\
205 \text { sec. } \\
227 \text { sec. }\end{array}$ \\
\hline
\end{tabular}

TABLE 4.3

Finite element discretization

\begin{tabular}{|r|r|r|r|r|}
\hline$\tau$ & JD & Arnoldi & Hwang et al. & precond. \\
\hline 0.1 & 261.4 & 1084.1 & 1212.4 & 3.4 \\
0.01 & 132.7 & 117.1 & 155.7 & 71.7 \\
0.001 & 118.9 & 61.2 & 96.0 & 246.6 \\
0.0001 & 155.6 & 46.6 & 71.1 & 665.6 \\
\hline
\end{tabular}

5. Conclusions. We discussed iterative projection methods of Arnoldi and Jacobi-Davidson type (including a variant proposed by Hwang et al.) for determining a few electronic states of a three-dimensional quantum dot taking into account an electron effective mass which depends on the position and on the electron energy level. Taking advantage of the symmetry of the governing rational eigenproblem it can be solved efficiently by both methods. If an accurate preconditioner is at hand the Arnoldi method is faster than Jacobi-Davidson type methods, however, the JacobiDavidson method seems to be more robust with respect to coarse preconditioners.

\section{REFERENCES}

[1] G. Bastard. Wave Mechanics Applied to Semiconductor Heterostructures. Les editions de physique, Les Ulis Cedex, 1988.

[2] T. Betcke and H. Voss. A Jacobi-Davidson-type projection method for nonlinear eigenvalue problems. Future Generation Computer Systems, 20(3):363 - 372, 2004.

[3] S.L. Chuang. Physics of Optoelectronic Devices. John Wiley \& Sons, New York, 1995.

[4] FEMLAB, Version 3.1. COMSOL, Inc., Burlington, MA, USA, 2004

[5] K. P. Hadeler. Variationsprinzipien bei nichtlinearen Eigenwertaufgaben. Arch.Rat.Mech.Anal., 30:297 - 307, 1968 .

[6] P. Harrison. Quantum Wells, Wires and Dots. Theoretical and Computational Physics. John Wiley \& Sons, Chicester, 2000.

[7] T.-M. Hwang, W.-W. Lin, J.-L. Liu, and W. Wang. Jacobi-Davidson methods for cubic eigenvalue problems. Numer.Lin.Alg.Appl., 12:605 - 624, 2005.

[8] T.-M. Hwang, W.-W. Lin, W.-C. Wang, and W. Wang. Numerical computation for pyramid quantum dot. In P. Neittaanmäki, T. Rossi, S. Korotov, E. Onate, J. Periaux, and D. Knörzer, editors, Proceedings of the European Congress on Computational Methods in Applied Sciences and Engineering. ECCOMAS 2004, Jyväskylä, Finland, 2004. ISBN 951-39-1869-6.

[9] T.-M. Hwang, W.-W. Lin, W.-C. Wang, and W. Wang. Numerical simulation of three dimensional quantum dot. J. Comput.Phys., 196:208 - 232, 2004.

[10] Y. Li. Numerical calculation of electronic structure for three-dimensional nanoscale semiconductor quantum dots and rings. J. Comput. Electronics, 2:49 - 57, 2003.

[11] Y. Li, O. Voskoboynikov, C.P. Lee, and S.M. Sze. Computer simulation of electron energy level for different shape InAs/GaAs semiconductor quantum dots. Comput.Phys.Comm., 141:66 $-72,2001$.

[12] Y. Li, O. Voskoboynikov, C.P. Lee, S.M. Sze, and O. Tretyak. Electron energy state dependence on the shape and size of semiconductor quantum dots. J.Appl.Phys., 90:6416 - 6420, 2001.

[13] K. Meerbergen. Locking and restarting quadratic eigenvalue solvers. SIAM J. Sci. Comput., 
$22: 1814-1839,2001$.

[14] A. Neumaier. Residual inverse iteration for the nonlinear eigenvalue problem. SIAM J. Numer. Anal., 22:914 - 923, 1985.

[15] G.L. Sleijpen, G.L. Booten, D.R. Fokkema, and H.A. van der Vorst. Jacobi-Davidson type methods for generalized eigenproblems and polynomial eigenproblems. BIT, 36:595 - 633, 1996.

[16] G.L. Sleijpen and H.A. van der Vorst. A Jacobi-Davidson iteration method for linear eigenvalue problems. SIAM J.Matr.Anal.Appl., 17:401 - 425, 1996.

[17] H. Voss. An Arnoldi method for nonlinear symmetric eigenvalue problems. In Online Proceedings of the SIAM Conference on Applied Linear Algebra, Williamsburg., http://www.siam.org/meetings/laa03/, 2003.

[18] H. Voss. Initializing iterative projection methods for rational symmetric eigenproblems. In Online Proceedings of the Dagstuhl Seminar Theoretical and Computational Aspects of Matrix Algorithms, Schloss Dagstuhl 2003, ftp://ftp.dagstuhl.de/pub/Proceedings/03/03421/03421.VoszHeinrich.Other.pdf, 2003.

[19] H. Voss. An Arnoldi method for nonlinear eigenvalue problems. BIT Numerical Mathematics, 44:387 - 401, 2004.

[20] H. Voss. A Jacobi-Davidson method for nonlinear eigenproblems. In M. Buback, G.D. van Albada, P.M.A. Sloot, and J.J. Dongarra, editors, Computational Science - ICCS 2004, 4th International Conference, Kraków, Poland, June 6-9,2004,Proceedings, Part II, volume 3037 of Lecture Notes in Computer Science, pages 34-41, Berlin, 2004. Springer Verlag.

[21] H. Voss. Electron energy level calculation for quantum dots. Technical Report 91, Institute of Mathematics, Hamburg University of Technology, 2005. Submitted to Comput. Phys. Comm.

[22] H. Voss and B. Werner. A minimax principle for nonlinear eigenvalue problems with applications to nonoverdamped systems. Math.Meth.Appl.Sci., 4:415-424, 1982.

[23] W. Wang, T.-M. Hwang, W.-W. Lin, and J.-L. Liu. Numerical methods for semiconductor heterostructures with band nonparabolicity. J.Comput.Phys., 190:141 - 158, 2003. 